\title{
Stability of Quasicrystals Composed of Soft Isotropic Particles
}

\author{
Kobi Barkan, ${ }^{1}$ Haim Diamant, ${ }^{2}$ and Ron Lifshitz ${ }^{1, *}$ \\ ${ }^{1}$ Raymond and Beverly Sackler School of Physics and Astronomy, Tel Aviv University, 69978 Tel Aviv, Israel \\ ${ }^{2}$ Raymond and Beverly Sackler School of Chemistry, Tel Aviv University, 69978 Tel Aviv, Israel
}

(Dated: March 15, 2011)

\begin{abstract}
Quasicrystals whose building blocks are of mesoscopic rather than atomic scale have recently been discovered in several soft-matter systems. Contrary to metallurgic quasicrystals whose source of stability remains a question of great debate to this day, we argue that the stability of certain soft-matter quasicrystals can be directly explained by examining a coarse-grained free energy for a system of soft isotropic particles. We show, both theoretically and numerically, that the stability can be attributed to the existence of two natural length scales in the pair potential, combined with effective three-body interactions arising from entropy. Our newly gained understanding of the stability of soft quasicrystals allows us to point at their region of stability in the phase diagram, and thereby may help control the self-assembly of quasicrystals and a variety of other desired structures in future experimental realizations.
\end{abstract}

PACS numbers: 61.44.Br, 64.70.M-, 64.75.Yz, 82.70.-y

Quasicrystals are more common than one had originally expected when their discovery was first announced!1 More than a hundred different metallic alloys are known to form stable quasicrystalline phases of icosahedral symmetry alone $\stackrel{2]}{,}$ with a few dozen additional stable phases exhibiting decagonal (10-fold) and possibly other symmetries. ${ }^{[3}$ Yet, to this date, there is no general agreement regarding the origin of their stability and the respective roles of energy and entropy in determining the observed phases. $\stackrel{4}{ }$ These growing numbers of stable solid-state quasicrystals, whose building blocks are on the atomic scale, have been joined in recent years by a host of soft-matter systems exhibiting quasiperiodic long-range order with building blocks on a much larger scale of tens to hundreds of nanometers - micelle-forming dendrimers ${ }^{[5] 6]}$ star block copolymers ${ }^{7}$ mesoporous silica, $\frac{8}{6}$ and binary systems of nanoparticles ${ }^{9}$ These newly discovered soft quasicrystals hold the promise for applications based on self-assembled nanomaterials, 10 with unique electronic or photonic properties that take advantage of their quasiperiodicity 11 At the same time, they provide alternative experimental platforms for the basic study of quasiperiodic longrange order, and offer the opportunity to study the thermodynamic stability of quasicrystals from a fresh viewpoint. To this date, soft quasicrystals have been observed only with dodecagonal point-group symmetry, having quasiperiodic order in the 12-fold plane and periodic order normal to the plane, whereas dodecagonal solid-state quasicrystals are rare and mostly only metastable ${ }^{3}$ Soft quasicrystals may belong, therefore, to a distinct class of quasicrystals, whose source of stability is likely to be different from their solid-state counterparts. We propose here a simple theoretical framework to address these new systems. We use it to explain the stability of the observed structures and indicate the (surprisingly simple) minimum conditions under which quasicrystals could be stabilized. Knowledge of these conditions gives us the ability to estimate the location of the region in the phase diagram where quasicrystals should be stable, and thus may help control the self-assembly of quasicrystals and other desired structures in future experimental realizations.

Several microscopic models have been studied over the years, mainly using computer simulations, but also using sophisticated analytical methods such as thermodynamic perturbation theory, ${ }^{12}$ to explore the structures arising from pair potentials that possess more than one microscopic length scale. These studies have yielded surprisingly rich phase diagrams even within the limited scope of single-component systems, interacting via isotropic pair potentials, 13,17 in some cases even finding stable quasicrystals 1218 On the other hand, phenomenological models based on coarse-grained free energies have been widely applied to treat phase diagrams and transitions ${ }^{22}$ and to explain the stability of different phases, including quasicrystals $\frac{23}{23}$ This is especially true in the case of soft-matter systems ${ }^{2425}$ due to their intermediate mesoscopic building blocks, which are significantly larger than the atomic scale, rendering a longwavelength gradient expansion a valid approximation.

A particular free energy of this sort, which is relevant for what follows below, was developed by Lifshitz and Petrich $\sqrt[26]{26}$ (henceforth LP) who extended the SwiftHohenberg equation ${ }^{27}$ to study parametrically-excited surface waves (Faraday waves) $\stackrel{28}{, 2}$ exhibiting dodecagonal quasiperiodic order. The LP free energy has the form

$$
\begin{aligned}
\mathcal{F}_{\mathrm{LP}}[\rho(\mathbf{r})]= & \int d x d y\left\{\frac{1}{2}\left[\left(\nabla^{2}+1\right)\left(\nabla^{2}+q^{2}\right) \rho\right]^{2}\right. \\
& \left.-\frac{1}{2} \varepsilon \rho^{2}-\frac{1}{3} \alpha \rho^{3}+\frac{1}{4} \rho^{4}\right\},
\end{aligned}
$$

where $\nabla^{2}=\partial_{x}{ }^{2}+\partial_{y}{ }^{2}$ is the 2-dimensional Laplacian. It is quite generic, imposing only two requirements on a material described by a 2 -dimensional density $\rho(x, y)$ : (a) the existence of two characteristic length scales, whose ratio is given by the parameter $q$; and (b) effective 3-body 
interactions, weighted by the parameter $\alpha$, that act to stabilize structures containing triplets of density modes with wave vectors adding up to zero. LP showed that if $q$ is chosen around $2 \cos (\pi / 12)=\sqrt{2+\sqrt{3}} \simeq 1.93$ one can obtain a quasiperiodic ground state with dodecagonal symmetry, yet no choice of $q$ yields globally-stable ground states with octagonal or decagonal symmetry, due to insufficiently-many resonant triplets of modes. Inspired by this simple result, we conjectured that the existence of two characteristic length scales along with 3-body interactions may constitute the source of stability of soft quasicrystals, all of which (to date) are dodecagonal. ${ }^{29}$ Here we confirm this conjecture by coarse-graining a microscopic partition function for isotropic soft particles into an effective free energy. In the limit of small deviations away from the uniform, or liquid, phase this coarse-grained free energy can be expanded in a power series and mapped onto the simple LP form (1), allowing us to gain important insight from the simpler LP model, and consequently to explain the stability of the observed phases using the full coarse-grained free energy.

Our starting point is the grand partition function for a system of particles with pairwise interactions,

$$
\begin{aligned}
& \mathcal{Z}=\sum_{N=0}^{\infty} \frac{e^{\beta \mu N}}{N !} \int \prod_{n=1}^{N} d \mathbf{r}_{n} e^{-\beta \mathcal{H}\left[\left\{\mathbf{r}_{n}\right\}\right]}, \\
& \mathcal{H}\left[\left\{\mathbf{r}_{n}\right\}\right]=\frac{1}{2} \sum_{m \neq n} U\left(\mathbf{r}_{m}-\mathbf{r}_{n}\right),
\end{aligned}
$$

where $\left\{\mathbf{r}_{n}\right\}_{n=1, \ldots, N}$ are the 2-dimensional positions of the centers of $N$ particles, $U$ their pair potential, $\beta=$ $\left(k_{\mathrm{B}} T\right)^{-1}$ the inverse temperature, and $\mu$ the chemical potential, which determines the mean particle density, $\bar{c}$. Using standard methods, $\stackrel{30}{ }$ one can rewrite the partition function in terms of collective coordinates - namely, the particle density, $c(\mathbf{r}) \equiv \sum_{n=1}^{N} \delta\left(\mathbf{r}-\mathbf{r}_{n}\right)$, and its conjugate field - rather than discrete positions. At the mean-field level, which amounts to a saddle-point approximation for the integration over the conjugate field, the transformed partition function becomes $\mathcal{Z}=\int \mathcal{D} c e^{-\beta \mathcal{F}[c]}$, where

$$
\begin{aligned}
\mathcal{F}[c(\mathbf{r})]= & \frac{1}{2} \int d \mathbf{r} d \mathbf{r}^{\prime} c(\mathbf{r}) U\left(\mathbf{r}-\mathbf{r}^{\prime}\right) c\left(\mathbf{r}^{\prime}\right) \\
& +\int d \mathbf{r}\left\{k_{\mathrm{B}} T c(\mathbf{r})[\ln c(\mathbf{r})-1]-\mu c(\mathbf{r})\right\} .
\end{aligned}
$$

The coarse-grained free energy functional given in Eq. (3) contains the familiar mean-field terms of pair interaction and ideal entropy. Although it could have been written from the outset, we wish to highlight the ability to extend the current theory to higher order, particularly in light of the cautionary remarks of Schwartz and Vinograd 31 We shall assume that the equilibrium density field is the one that minimizes $\mathcal{F}$ for the given $T$ and $\mu$ and the specific choice of $U(\mathbf{r})$. Results of such direct minimization will be presented shortly. However, since it is not a priori obvious what pair potentials and thermodynamic parameters may yield quasicrystalline order, it is beneficial first to characterize $\mathcal{F}$ and relate it to $\mathcal{F}_{\mathrm{LP}}$ of Eq. (1).
Above a certain critical temperature, $T>T_{\mathrm{c}}$, the equilibrium density for any $\mu$ should be uniform, $c(\mathbf{r}) \equiv \bar{c}$. Minimizing $\mathcal{F}$ with respect to such a uniform field yields the relation,

$$
\mu=k_{\mathrm{B}} T \ln \bar{c}+\tilde{U}_{0} \bar{c},
$$

where $\tilde{U}_{0} \equiv \int d \mathbf{r} U(\mathbf{r})$. For $T<T_{\mathrm{c}}$ the equilibrium density is expected to become nonuniform at a certain value of $\mu$ (or, alternatively, above a certain mean density $\bar{c}$ ). Assuming that $T$ is only slightly smaller than $T_{\mathrm{c}}$, we substitute $c(\mathbf{r})=\bar{c}[1+\rho(\mathbf{r})]$ in $\mathcal{F}$ and expand to 4 th order in small $\rho$. The result can be written as

$$
\begin{aligned}
& \frac{\mathcal{F}[\rho]}{\bar{c} k_{\mathrm{B}} T_{\mathrm{c}}} \simeq \int \frac{d \mathbf{k}}{(2 \pi)^{2}} \frac{1}{2} \frac{\tilde{U}(\mathbf{k})-\tilde{U}_{\min }}{\left|\tilde{U}_{\min }\right|}|\tilde{\rho}(\mathbf{k})|^{2} \\
& +\int d \mathbf{r}\left\{\frac{1}{2} \frac{T-T_{\mathrm{c}}}{T_{\mathrm{c}}} \rho^{2}(\mathbf{r})-\frac{1}{6} \frac{T}{T_{\mathrm{c}}} \rho^{3}(\mathbf{r})+\frac{1}{12} \frac{T}{T_{\mathrm{c}}} \rho^{4}(\mathbf{r})\right\},
\end{aligned}
$$

where tildes denote Fourier-transformed quantities. The critical temperature, below which perturbations start to grow, is given by

$$
k_{\mathrm{B}} T_{\mathrm{c}}=-\bar{c} \tilde{U}_{\min },
$$

where $\tilde{U}_{\min }$ is the minimum of the Fourier-transformed pair potential, which must be negative for $T_{\mathrm{c}}$ to be positive. The approximate coarse-grained free energy (5) resembles $\mathcal{F}_{\mathrm{LP}}(1)$, with the gradient term replaced by a Fourier-space integral of the pair potential, and the expansion in powers of the density arising from the entropy term of Eq. (3).

To try to predict the conditions under which dodecagonal quasicrystals minimize $\mathcal{F}$ of Eq. (3), let us recall the findings of LP, who showed that such crystals are stabilized in their simpler model, $\mathcal{F}_{\mathrm{LP}}$ of Eq. (1), under the following conditions: (i) $q \simeq 2 \cos (\pi / 12)$; (ii) $0<$ $\varepsilon / \alpha^{2} \lesssim 0.088$. LP further showed that, for $\varepsilon / \alpha^{2} \gtrsim 0.088$, a hexagonal state is obtained. In terms of our free energy expansion (5), condition (i) translates into a requirement for the properties of $\tilde{U}(\mathbf{k})$. The first two minima of this function should be located at wavevectors $k_{1}$ and $k_{2}$, whose ratio $q=k_{2} / k_{1}$ is around $2 \cos (\pi / 12) \simeq 1.93$, and should have a similar depth. Condition (ii) translates into a requirement for the thermodynamic parameters, $T$ and $\mu$ (or $\bar{c}$ ), namely, $0<1-T / T_{\mathrm{c}} \lesssim 0.066$, i.e. $T$ is restricted to a small range below $T_{\mathrm{c}}$.

We test these estimates, obtained from the approximate free energy expansion (5), against the numerical minimization of $\mathcal{F}[c(\mathbf{r})]$ of Eq. (3). We find that a variety of isotropic pair potentials, three examples of which are shown in Fig. 1, contain a sufficient number of tunable parameters to satisfy condition (i) above. The form of these potentials is motivated by the qualitative features of the experimental system, $\frac{5}{5}$ consisting of spherical micelles whose interaction should contain an inner repulsive core, a region governed by van der Waals attraction, and a longer-range soft repulsion. Using these potentials in Eq. (3), we find the minimum free-energy 

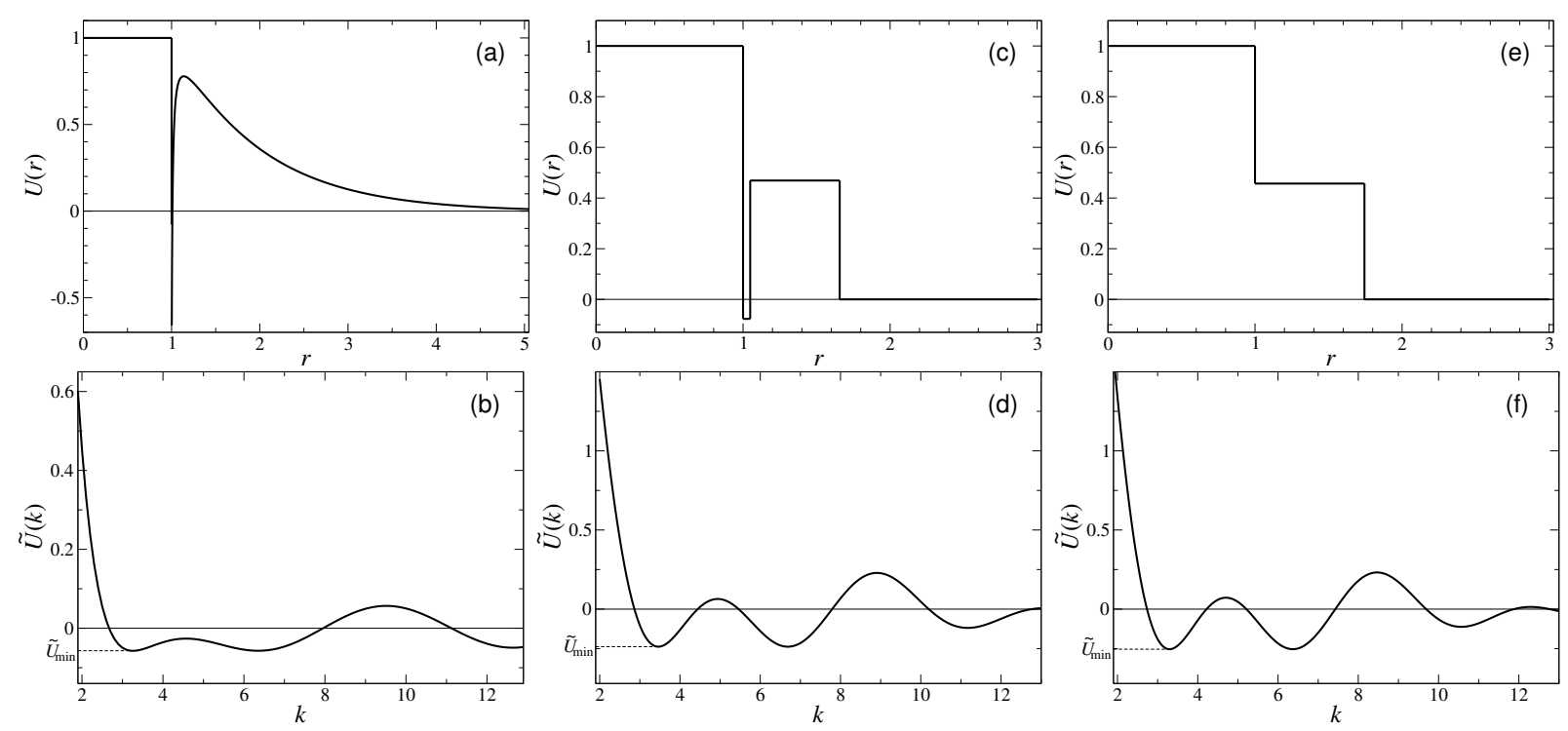

FIG. 1. Three of the isotropic pair-potentials used in this study (top row), whose Fourier transforms (bottom row) are designed so that the ratio $q$ of the positions of their first two minima is approximately 1.93. The first potential in (a), motivated by the structure of dendritic micelles, consists of a normalized repulsive core, with a short range (van der Waals) attraction at the core boundary, and an exponentially-decaying soft shoulder. This behavior is approximated by even simpler 3 -step and 2-step potentials, shown in (c) and (e). Potential parameters: (a) $U(r)=1$ for $0<r<1$; and $-u_{1} /(r-1+\delta)+u_{2} \exp [-(r-1) / \lambda]$, with $u_{1}=0.02057, u_{2}=1.057, \lambda=0.9756$, and $\delta=0.01$, for $r>1$. (c) $U(r)=1$ for $0<r<1 ;-0.0770$ for $1<r<1.0485$; 0.4690 for $1.0485<r<1.6570$; and 0 for $r>1.6570$. (e) $U(r)=1$ for $0<r<1$; 0.457 for $1<r<1.7442$; and 0 for $r>1.7442$.

state by numerically integrating the corresponding relaxational equation, $\partial_{t} c=-\delta \mathcal{F} / \delta c$, using a pseudospectral method - with local terms evaluated in direct space and non-local terms in Fourier space - starting with random initial conditions and waiting until a steady state is obtained. We note that this equation does not describe the actual dynamics and is used merely as a minimization tool.

When $q$ is selected according to condition (i) - as demonstrated in Fig. 1 and $T$ and $\mu$ are set such that $T$ is just below $T_{\mathrm{c}}$ [condition (ii)], we indeed find a minimum free-energy state which is a dodecagonal quasicrystal, as shown on the left-hand column of Fig. 2 for the particular case of the 3-step potential of Fig. 1(c). When $T$ is decreased slightly further a transition is observed to a hexagonal state [middle column of Fig. 2, restricting the stability of dodecagonal quasicrystals to a narrower range than that predicted by the approximate free energy expansion (5) ${ }^{32}$ The same stabilizing mechanism of two length scales and 3-body interactions can be used to obtain other structures, $\underset{26]}{ }$ for example, setting $q=\sqrt{3}$ yields immediately below $T_{\mathrm{c}}$ a hexagonal crystal, shown on the right-hand column of Fig. 2. We have observed that slight variations in the potential parameters still yield the expected phases. A systematic study of the stability boundaries in parameter space is left for future work, perhaps using more realistic potentials, derived from specific experimental realizations.

While the first two minima of the Fourier-transformed pair potential $\tilde{U}(k)$ must be negative to give a positive
$T_{\mathrm{c}}$, the value of $\tilde{U}(k=0)$ need not be negative. This allows a purely repulsive pair potential $U(r)$ to stabilize a quasicrystal, as we show here for the simple two-step potential of Fig. 1(e). Despite the appealing simplicity of this potential - defined after scaling by two parameters only - we emphasize that potentials of this sort can satisfy the requirements on the Fourier space minima only near a single choice of their two parameters. As a consequence, attempting a naïve numerical search for a dodecagonal quasicrystal with this potential, by setting the extent of the second step to $R=2 \cos (\pi / 12) \simeq 1.93$, would fail. We now understand that in order for nonlinear mode interactions to stabilize a dodecagonal quasicrystal, one must adjust this ratio not in the real-space pair potential, but rather in its Fourier transform. This is obtained in this case by setting $R \simeq 1.74$ in real space. We have managed to stabilize a dodecagonal quasicrystal with the simple two-step potential by eliminating the need to search around in parameter space. Our theoretical understanding of the source of stability allows us to point at the stability regions in parameter space, even when these regions are extremely narrow.

Thus, we confirm that the existence of two characteristic length scales and sufficiently strong 3-body or higher-order nonlinear interactions can account for the stability of dodecagonal quasicrystals of isotropic soft particles. More specifically, we determine the two length scales through requirements on the minima of the Fourier-transformed pair potential and, although 3-body terms may arise from various interactions, $\frac{[33}{,}$ we show 


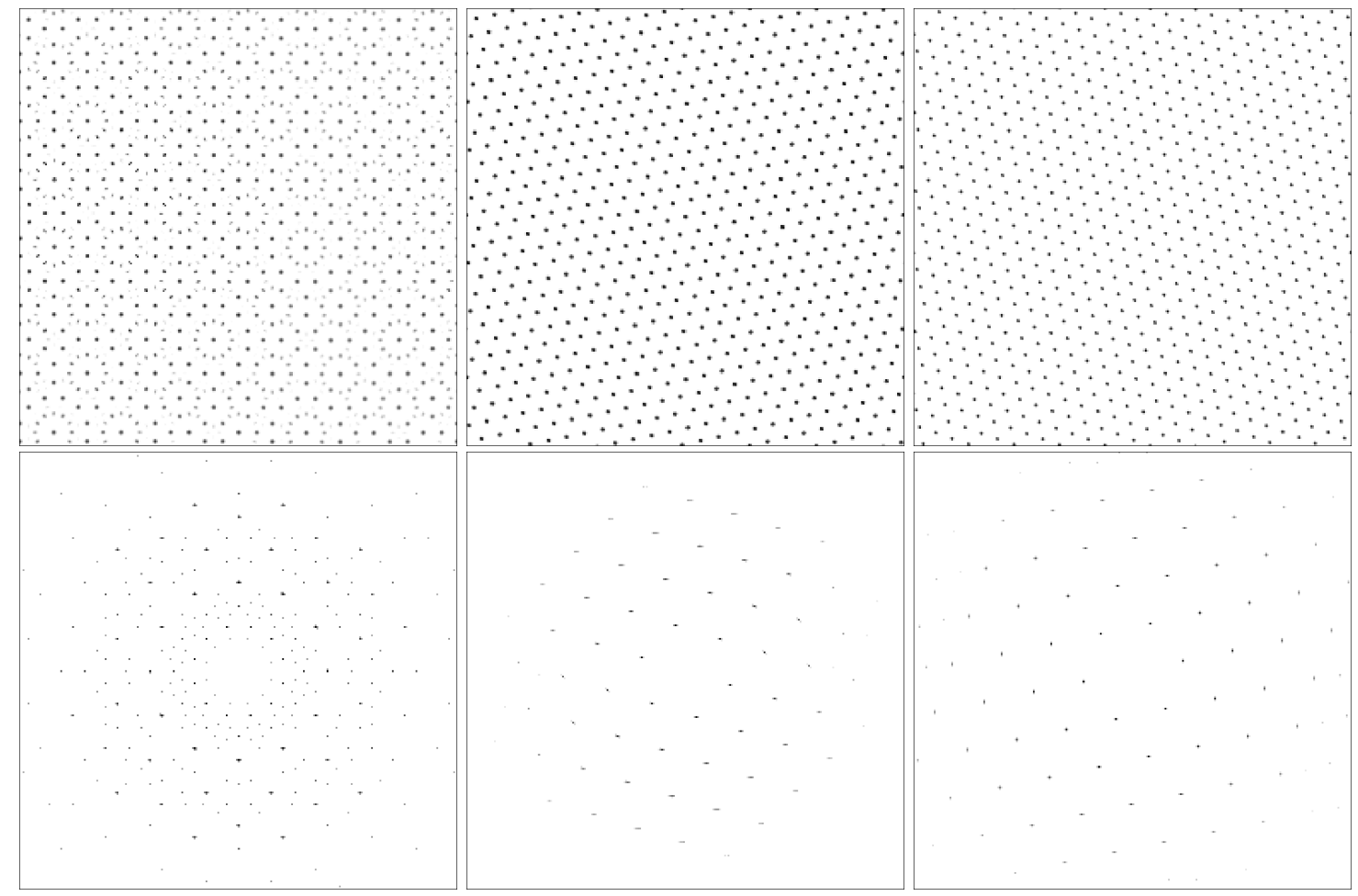

FIG. 2. Real space densities $c(\mathbf{r})$ (top row) and their Fourier transforms $|c(\mathbf{k})|$, with $c(\mathbf{k}=0)$ removed (bottom row), obtained by a numerical minimization of the free energy (3), with the 3-step potential of Fig. 1 . Other potentials yield similar results. Dodecagonal crystal (left column): 3-step potential, with parameters specified in Fig. 1(c). Thermodynamic variables are $\mu=2.2895$ and $T=0.999 T_{\mathrm{c}}$. Hexagonal crystal (middle column): Same as in the left column, but with a lower temperature $T=0.980 T_{\mathrm{c}}$. Hexagonal crystal (right column): 3 -step potential similar to Fig. 1(c), with parameters $U(r)=1$ for $0<r<1$; -0.115 for $1<r<1.053 ; 0.370$ for $1.053<r<1.600$; and 0 for $r>1.600$, designed so that $q \simeq \sqrt{3}$. Thermodynamic variables are $\mu=1.9041$ and $T=0.999 T_{\mathrm{c}}$.

that translational entropy suffices to provide the required term. Thus, the delicate interplay between interaction and entropy can give rise to stable quasicrystals even for relatively simple isotropic potentials. By designing these potentials along the guidelines provided here, one should be able to control the self-assembly of quite complex structures. Preliminary tests, using molecular dynamics simulations, indicate that our design principles seem to work ${ }^{34}$

This work can be extended in several directions. The results of the approximate theory presented here should be verified using direct computer simulations. Importantly, such simulations can be used to clarify the actual stability regions of the quasicrystalline phase. The dynamics of crystallization, and of collective degrees of freedom in the ordered state, can be studied by replacing the equation used here to minimize the free energy by one that is adequate for a conserved density field, possibly while taking thermal fluctuations into account. Finally, a similar coarse-graining procedure could be applied to two-component systems or anisotropic potentials!79

We are grateful to Michael Cross, Michael Engel, and Moshe Schwartz for fruitful discussions. This research is supported by the Israel Science Foundation through Grants No. 684/06 and 556/10.
* Corresponding author: ronlif@tau.ac.il

1 D. Shechtman, I. Blech, D. Gratias, and J. W. Cahn, Phys. Rev. Lett., 53, 1951 (1984).

${ }^{2}$ A. P. Tsai, Sci. Technol. Adv. Mater., 9, 013008 (2008).

3 W. Steurer, Z. Kristallogr., 219, 391 (2004)

4 M. de Boissieu, Phil. Mag., 86, 1115 (2006); C. L. Henley, ibid., 86, 1123 (2006)

5 X. Zeng, G. Ungar, Y. Liu, V. Percec, A. E. Dulcey, and
J. K. Hobbs, Nature, 428, 157 (2004) X. Zeng, Current Opin. Colloid Interface Sci., 9, 384 (2005)

o V. Percec, M. R. Imam, M. Peterca, D. A. Wilson, and P. A. Heiney, J. Am. Chem. Soc., 131, 1294 (2009), V. Percec, M. R. Imam, M. Peterca, D. A. Wilson, R. Graf, H. W. Spiess, V. S. K. Balagurusamy, and P. A. Heiney, ibid., 131, 7662 (2009).

7 A. Takano, W. Kawashima, A. Noro, Y. Isono, N. Tanaka, 
T. Dotera, and Y. Matsushita, J. Polym. Sci. Polym. Phys., 43, 2427 (2005); K. Hayashida, T. Dotera, A. Takano, and Y. Matsushita, Phys. Rev. Lett., 98, 195502 (2007)

${ }^{8}$ C. Xiao, K. Miyasaka, N. Fujita, Y. Sakamoto, and O. Terasaki, in ICQ11-Book of Abstracts, Y. Ishii and T. Ishimasa, eds. (Hokkaido University, Sapporo, 2010) p. 28. Reproduced with permission as figure 2 of J. M. Dubois and R. Lifshitz, Phil. Mag., In press (2011).

9 D. V. Talapin, E. V. Shevchenko, M. I. Bodnarchuk, J. C. X. Ye, and C. B. Murray, Nature, 461, 964 (2009).

10 V. Percec, M. Glodde, T. K. Bera, Y. Miura, I. Shiyanovskaya, K. D. Singer, V. S. K. Balagurusamy, P. A. Heiney, I. Schnell, A. Rapp, H.-W. Spiess, S. D. Hudson, and H. Duan, Nature, 419, 384 (2002); D. K. Smith, A. R. Hirst, C. S. Love, J. G. Hardy, S. V. Brignell, and B. Huang, Prog. Polym. Sci., 30, 220 (2005)

11 R. Lifshitz, in Silicon Versus Carbon, edited by Y. Magarshak, S. Kozyrev, and A. K. Vaseashta (Springer, Dordrecht, 2009) pp. 119-136.

12 A. R. Denton and H. Löwen, Phys. Rev. Lett., 81, 469 (1998)

13 E. A. Jagla, J. Chem. Phys., 110, 451 (1999)

14 P. Ziherl and R. D. Kamien, J. Phys. Chem. B, 105, 10147 (2001) M. A. Glaser, G. M. Grason, R. D. Kamien, A. Košmrlj, C. D. Santangelo, and P. Ziherl, Europhys. Lett., 78, 46004 (2007).

io G. J. Pauschenwein and G. Kahl, J. Chem. Phys., 129, 174107 (2008)

10 F. Saija, S. Prestipino, and G. Malescio, Phys. Rev. E, 80, 031502 (2009) S. Prestipino, F. Saija, and G. Malescio, Soft Matter, 5, 2795 (2009).

17 H. Shin, G. M. Garson, and C. D. Santangelo, Soft Matter, 5, 3629 (2009)

18 M. Dzugutov, Phys. Rev. Lett., 70, 2924 (1993).

19 A. Quandt and M. P. Teter, Phys. Rev. B, 59, 8586 (1999) A. Skibinsky, S. V. Buldyrev, A. Scala, S. Havlin, and
H. E. Stanley, Phys. Rev. E, 60, 2664 (1999)

${ }^{20}$ M. Engel and H.-R. Trebin, Phys. Rev. Lett., 98, 225505 (2007) Z. Kristallogr., 223, 721 (2008).

${ }^{21}$ A. S. Keys and S. C. Glotzer, Phys. Rev. Lett., 99, 235503 (2007)

22 S. Alexander and J. McTague, Phys. Rev. Lett., 41, 702 (1978)

${ }^{23}$ P. A. Kalugin, A. Y. Kitaev, and L. C. Levitov, JETP Lett., 41, 145 (1985); N. D. Mermin and S. M. Troian, Phys. Rev. Lett., 54, 1524 (1985); L. Gronlund and N. D. Mermin, Phys. Rev. B, 38, 3699 (1988)

24 G. Gompper and M. Schick, Self-Assembling Amphiphilic Systems, edited by C. Domb and J. L. Lebowitz, Phase Transitions \& Critical Phenomena, Vol. 16 (Academic Press, London, 1994).

25 T. Dotera and T. Gemma, Phil. Mag., 86, 1085 (2006) T. Dotera, 87, 3011 (2007)

${ }^{26}$ R. Lifshitz and D. M. Petrich, Phys. Rev. Lett., 79, 1261 (1997)

27 J. B. Swift and P. C. Hohenberg, Phys. Rev. A, 15, 319 (1977) M. Cross and H. Greenside, "Pattern formation and dynamics in nonequilibrium systems," (Cambridge University Press, Cambridge, 2009) Chap. 5.

28 W. S. Edwards and S. Fauve, Phys. Rev. E, 47, R788 (1993)

${ }_{29}$ R. Lifshitz and H. Diamant, Phil. Mag., 87, 3021 (2007).

${ }^{30}$ G. H. Fredrickson, The equilibrium theory of inhomogeneous polymers (Oxford University Press, Oxford, 2006).

31 M. Schwartz and G. Vinograd, Physica A, 308, 227 (2002)

32 This narrower stability region, however, may be a consequence of our mean-field coarse-graining and should be checked in a more detailed future study.

33 C. von Ferber, A. Jusufi, C. N. Likos, H. Löwen, and M. Watzlawek, Eur. Phys. J. E, 2, 311 (2000).

34 Michael Engel, private communication. 